\title{
Combinational Test Generation for Acyclic Sequential Circuits using a Balanced ATPG Model*
}

\author{
Yong Chang $\mathrm{Kim}^{\dagger}$ \\ University of Wisconsin-Madison \\ Madison, WI 53706 USA \\ kimy@ece.wisc.edu
}

\author{
Vishwani D. Agrawal \\ Bell Labs, Lucent Tech. \\ Murray Hill, NJ 07974 USA \\ va@research.bell-labs.com
}

\author{
Kewal K. Saluja \\ University of Wisconsin-Madison \\ Madison, WI 53706 USA \\ saluja@engr.wisc.edu
}

\begin{abstract}
To create a combinational ATPG model for an acyclic sequential circuit, all unbalanced fanouts, i.e., fanouts reconverging with different sequential depths, are moved toward primary inputs using a retiming-like transformation. All flipflops are then shorted and unbalanced primary input fanouts are split as additional primary inputs. A combinational test vector for a fault in this model is converted into a vector sequence that detects the corresponding fault in the original circuit. An analysis classifies the undetected faults in this model as either untestable or multiply-testable. The latter, typically less than $5 \%$ of all faults, are modeled as special single faults in the combinational model. This procedure correctly treats various types of faults, namely, (a) faults detectable by repeating a pattern, (b) faults only detectable by non-repeated patterns, (c) faults only testable as multiple faults in the combinational model, and (d) sequentially undetectable faults. ISCAS ' 89 benchmark results verify that the given procedure achieves identical fault coverage and efficiency as a sequential ATPG and uses less CPU time.
\end{abstract}

\section{Introduction}

Two commonly used design for testability (DFT) methods are full scan [11] and partial scan [2]. In full scan, we scan all flip-flops (FFs) and use combinational automatic test pattern generation (ATPG). In partial scan, a subset of FFs is scanned. Thus, hardware overhead and testing time are reduced over full scan. However, one must use a sequential ATPG program whose complexity is significantly greater than that of combinational ATPG used in the full scan design.

Cheng and Agrawal $[3,8]$ and Kunzmann and Wunderlich [17] have proposed partial scan methods that break cycles to make the sequential structure acyclic in the test mode. An acyclic circuit has a finite maximum sequential depth $(d)$, defined as the largest number of FFs on any path between

\footnotetext{
*This work was supported in part by the National Science Foundation grant MIP9714034.

${ }^{\dagger}$ Summer Intern (1998 and 1999) at Bell Labs, Lucent Technologies, Murray Hill, NJ 07974.
}

primary inputs and outputs. Thus, using the time-frame expansion procedure [1], any fault can be tested by combinational ATPG applied to a circuit obtained by cascading the combinational logic $d$ times. Miczo [19] proposes the conversion of an acyclic circuit to a combinational model by copying the entire fanin cone of each FF. Min and Rogers [20] use a combinational ATPG model in which all FFs are shorted. They repeat the same vector $(d+1)$ times, but any undetected faults require sequential ATPG to achieve $100 \%$ fault efficiency. Kunzmann and Wunderlich [17, 22] propose a combinational ATPG model capable of generating both repeated and non-repeated vectors.

Besides being acyclic, a sequential circuit can have other structural properties that facilitate ATPG. A circuit is called a balanced circuit [15] if it is acyclic and all paths between any pair of nodes have the same number of FFs, where a node can be a primary input (PI), gate, FF or primary output (PO). The number of FFs on a path is referred to as the sequential depth of that path. Gupta et al. [15] propose a partial scan technique in which scan and other hardware are added to make the circuit balanced and testable via combinational ATPG.

Balakrishnan and Chakradhar [6] define strongly balanced circuits, a subclass of balanced circuits, in which all paths between PI and PO have the same sequential depth. They use combinational ATPG to derive tests that are significantly more compact than the partial scan sequences of the previous method [15]. Balakrishnan and Chakradhar [5] also propose a software transformation procedure to reduce (but not completely eliminate) the number of FFs in the ATPG model. That method, however, requires sequential ATPG since the ATPG model may contain FFs.

According to Fujiwara et al. $[12,13,14,16,21]$ a circuit that appears balanced when we ignore the PI nodes is called an internally balanced circuit. They use combinational ATPG by temporarily splitting the PI fanouts causing unbalance into separate PIs. Test sequences are then constructed for the internally balanced circuit, which requires less DFT overhead than a balanced circuit.

The present work is closely related to the proposal of Kunzmann and Wunderlich $[17,22]$. We give algorithms to produce the smallest combinational ATPG model for any general 


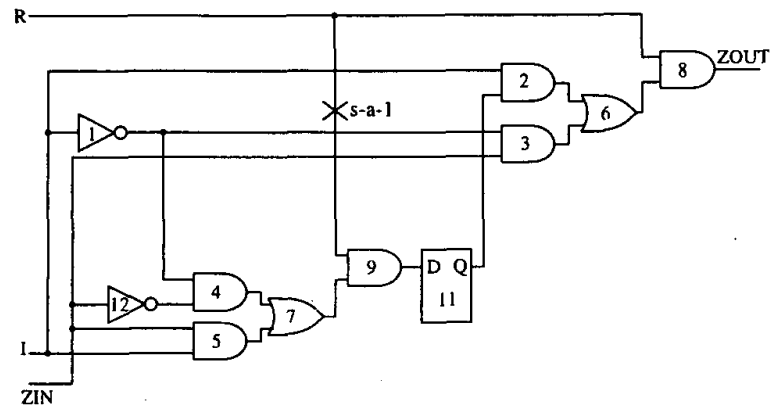

Figure 1. A single output circuit.

acyclic circuit (which does not have to be balanced), requiring no hardware modification in the original circuit. Because of splitting of signals, certain faults of the original circuit map onto multiple faults. Only in cases, where such multiple faults mask each other, we generate multiple fault tests. Our test generation system correctly deals with such situations and obtains a $100 \%$ fault efficiency. We assume that the given sequential circuit is synchronous and acyclic. The technique is also applicable to other circuits via partial scan [8] or resynthesis [9].

\section{Test Generation Approach}

Our test generation method has three steps, combinational ATPG model generation, combinational test generation and test transformation.

\subsection{Combinational ATPG model generation}

Gupta et al. [15] have defined a balanced sequential structure.

Definition 1 A synchronous sequential circuit is said to be balanced if it is acyclic and for any pair of signal nodes ( $P I$, $F F$, gates and $P O) v_{1}$ and $v_{2}$ in the circuit all directed paths (if any) from $v_{1}$ to $v_{2}$ are of equal sequential weight (depth). The weight or depth of a path is the number of FFs on it.

Consider the circuit in Figure 1. It is acyclic but not balanced. Notice that two paths between nodes $R$ and $Z O U T$ (AND gate 8 ) have sequential depths of 0 and 1 , respectively. Thus, PI $R$ can supply different values to the inputs of gate 8 via the two paths. In order to allow combinational ATPG to assign multiple values to a signal like $R$, we generate a balanced combinational circuit (BCC) model.

\section{Algorithm 1 BCC Generation}

- Levelization: In a single pass, starting from PIs (Pls have 0 weight), assign weights to all $P O$ nodes, where a $P O$ weight is the maximum number of FFs on any path between the PO and all reachable PIs. The weight of $a$ node $g$ is written as $w(g)$. Initialize all non-PO nodes to unassigned weights.

- Balancing: A node is balancea' if all of its fanout nodes with assigned weights have the same weight. Recursively, starting at POs, determine weights for all reachable node until every nodes in the circuit is balanced and there is no node with unassigned weight. Two cases occur as non-PO nodes $g_{i}$ are repeatedly processed:

Case 1 Node $g_{i}$ either has a single fanout node $g_{j}$ with assigned weight $w\left(g_{j}\right)$, or has multiple fanout nodes among which all nodes with assigned weight have the same value $w\left(g_{j}\right)$. Then, $w\left(g_{i}\right)=w\left(g_{j}\right)$, if $g_{i}$ is a combinational gate or $\mathrm{Pl}$, or $w\left(g_{i}\right)=$ $w\left(g_{j}\right)-1$, if $g_{i}$ is a FF.

Case 2 Node $g_{i}$ has fanouts to multiple nodes of which $n, g_{j q}, 1 \leq q \leq n$, have assigned weights, $w\left(g_{j q}\right)$. Suppose there are $m, m \leq n$, distinctly different values among these $n$ weights. Then, node $g_{i}$ is duplicated as $m$ nodes, $g_{i 1}$ through $g_{i m}$, each of the same type (i.e., PI, gate or FF) as $g_{i}$. If $g_{i}$ is a $P I$, then duplication creates $m-1$ new PIs. Otherwise, inputs to the duplicated nodes are supplied by adding $m-1$ new fancuts to each fanin node of $g_{i}$. A duplicated node $g_{i p}, 1 \leq p \leq m$, fans out to all those nodes among $g_{j q}, 1 \leq q \leq n$, that have the same weight. This transformation is known as duplicate and split (DAS). Next, the weights of the duplicated nodes are determined by Case 1.

- Combinationalization: Short all FFs or replace FFs with buffers to get the $B C C$.

Algorithm 1 assigns a weight to every node reachable from one or more POs. The recursion over POs leaves each node with one unique weight. Case 1 determines the weight of a balanced node. Case 2 deals with unbalanced nodes. The duplicate and split (DAS) procedure basically moves unbalanced fanouts one level backward (toward PIs). It leaves the function of the circuit unchanged if a PI and its split copies assume the same signal value. Successive application of DAS eventually moves all unbalances to l?Is, whose splitting creates a perfectly balanced structure.

The circuit in Figure 2 (a) illustrates the DAS transformation. The figure shows a portion of a circuit that is to be balanced. The weight of node $g_{k}$ is $w\left(g_{k}\right)$. Using Case 1 of Algorithm 1, the weight of node $g_{f}$ is found to be $w\left(g_{k}\right)-1$. The node $g_{j}$ has two fanouts that reconverge at $g_{k}$. These fanouts are unbalanced since the fanout nodes, $g_{k}$ and $g_{f}$, have different weights, $w\left(g_{k}\right)$ and $w\left(g_{k}\right)-1$, respectively. Applying Case 2 of Algorithm 1, we duplicate $g_{j}$ as $g_{j 1}$ and $g_{j 2}$, and split the fanouts so $g_{j 1}$ and $g_{j 2}$ have one fanout each to $g_{k}$ and $g_{f}$, respectively. We now apply Case 1 of Algorithm 1 , to assign weights, $w\left(g_{k}\right)$ to $g_{j 2}$ and $w\left(g_{k}\right)-1$ to $g_{j 1}$. 


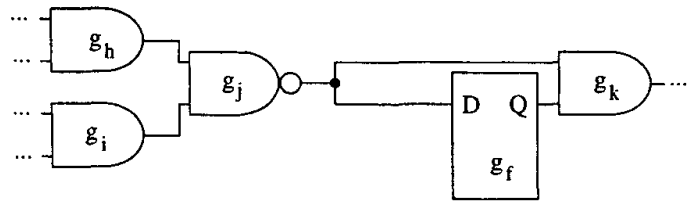

(a) Unbalanced multiple fanouts due to reconvergence

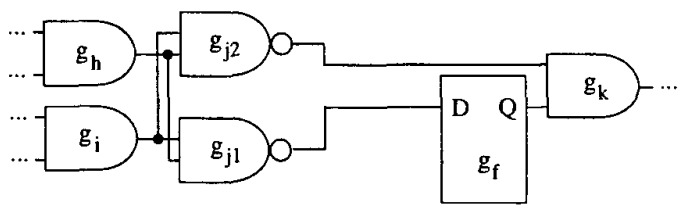

(b) Duplicate and split operation on $\mathrm{g}_{\mathrm{j}}$

Figure 2. A duplicate and split (DAS) example.

Notice, nodes $g_{h}$ and $g_{i}$ are now unbalanced and we will need to apply DAS to them. Figure 2 (b) shows the DAS transformation on $g_{j}$, which moved unbalanced fanouts from $g_{j}$ to the fanin nodes, $g_{h}$ and $g_{i}$. The use of DAS provides separate signal paths in the combinational model so that the copies of a signal may assume different values in different time frames. The recursive DAS transformation may create a circuit with new PIs having specific weights. When DAS is applied to all gates recursively, all unbalancedness is moved to PIs.

This circuit-transformation is similar to retiming [18]. However, retiming proposed by Leiserson and Saxe [18] cannot always be performed directly to the multiple fanout point. Using DAS, we can apply the retiming to move all unbalanced fanouts to PIs.

As we see from Figure 2 (b), the DAS operation on nonPI vertices will not alter the functional behavior of the circuit. Thus, the resultant circuit is functionally equivalent to the original one after recursive DAS transformations are performed only on internal fanouts and PI vertices are not modified. However, a single fault before the modification may map to multiple locations if it was duplicated. In a sequential circuit, an unbalanced fanout node may supply different values to branches during different time frames. To model such unbalanced fanout nodes, we modify the circuit by adding PIs that represent input values for different time frames. The effect of a single fault being repeated in multiple time frames is modeled as multiple faults in the duplicated logic of the combinational model.

Figure 3 shows the BCC of the circuit in Figure 1, where the FF is replaced with a buffer. As is evident from the figure, $R, I, Z I N$ and gate 1 have unbalanced fanouts. These PIs and the gate with unbalanced fanouts must be balanced using Algorithm 1. The multiple fanouts of node 1 are unbalanced since they reconverge at node 6 with different sequential depths. Thus, 1 is duplicated and split by Algorithm 1 .

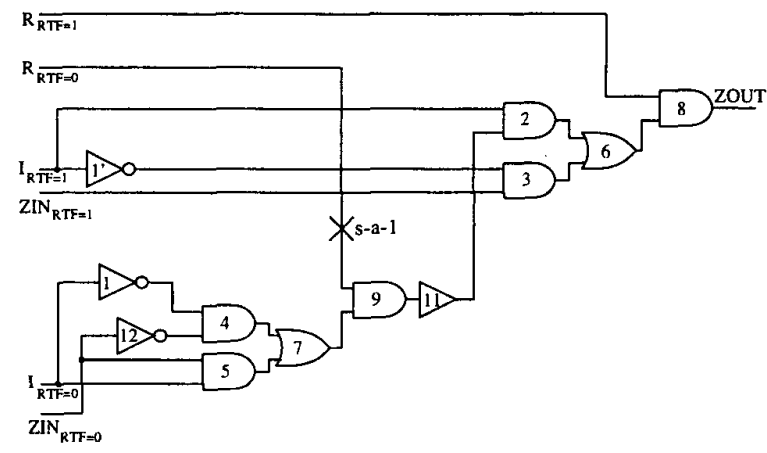

Figure 3. BCC for the circuit in Figure 1.

For obtaining the results of Section 3, the algorithm of this subsection was implemented by representing the circuit as a weighted directed acyclic graph (DAG). The vertices of this DAG are PIs, gates and POs. An arc between a vertex-pair represents a signal flow path and the integer weight of the arc equals the number of FFs on the path.

\subsection{Combinational Test-Generation}

A single fault in the original circuit maps onto a set of multiple faults or a single fault in the BCC depending upon whether or not the target fault site has been duplicated and split. The fault mapping algorithm is given below:

\section{Algorithm 2 Fault Mapping}

- Single fault mapping: $A$ fault in the original circuit maps onto a single fault in BCC if there is no duplicated node at the corresponding fault site in the $B C C$.

- Multiple fault mapping: A single fault of the original circuit maps onto a set of multiple faults if DAS transformation has created more than one copy of the fault site in the BCC. All copied lines and the original line are then the sites of simultaneously occurring single faults in the $B C C$.

If there is a test for a mapped single fault in the $\mathrm{BCC}$, then the fault will be detected in the sequential circuit by the sequentialized BCC test (see Section 2.3). Otherwise, the fault is undetectable. Similarly, if there is a test for a mapped multiple fault in the $\mathrm{BCC}$, then fault is detectable in the sequential circuit. Otherwise, the fault is undetectable.

The multiple fault mapping is a sufficient condition to detect the "original" fault, but it is not a necessary condition. If we generate a test using BCC for single faults on each multiple fault site, then some of those tests may detect the fault in the sequential circuit. However, such single fault assumption for a multiple fault does not guarantee the detection and the multiple fault model may be necessary for some faults. As we observe in Section 3, only a small fraction of faults 
needs to be modeled as multiple faults in the BCC. Once that is done, a $100 \%$ fault efficiency can be achieved by combinational ATPG.

\subsection{Test Transformation}

Let $d_{\max }$ denote the maximum sequential depth of the original sequential circuit. Weights assigned to PIs in the $\mathrm{BCC}$ are called relative time frames (RTFs). RTFs determine the time sequence of signal values at the PI. For example, $Z I N$ of the circuit shown in Figure 1 has unbalanced fanout branches and the corresponding $\mathrm{BCC}$ in Figure 3 has $R T F s$ of 0 and 1 . Using the RTF information, we can determine how to convert a combinational test vector of $\mathrm{BCC}$ to a sequential test. Each combinational vector produces a sequence of vectors of length up to $d_{\max }+1$. In general, the vector sequence length can be less than or equal to $d_{\max }+1$, since we only need to propagate the effect of the fault to an observable PO. We transform the combinational test vector generated for $\mathrm{BCC}$ to a vector sequence for the original circuit as follows:

Algorithm 3 Test-Transformation: Suppose that the BCC has $k+1$ copies of a PI node $x$ with RTFs of $\left\{t_{0}, t_{1}, \ldots, t_{k}\right\}$, $k \leq d_{\text {max }}$, sorted in ascending order, and the values on these $k+1$ copies in a combinational test derived for BCC are $\left\{v_{0}, v_{1}, \ldots, v_{k}\right\}$. The value of the PI $x$ in the $t_{i}$ th vector of the sequence is $v_{i}$. If some value $t_{j}, 0 \leq j \leq k$, does not occur among the RTFs of $x$, then a random value $(0$ or 1$)$ is assigned to the PI $x$ in the $t_{j}$ th vector.

Consider a stuck-at-1 fault on input $\mathrm{R}$ of gate 9 as shown in Figure 1. The corresponding fault in the BCC is shown in Figure 3. Using $\mathrm{BCC}$, the test is found to be $\left\{R_{R T F=0}, I_{R T F=0}, Z I N_{R T F=0}\right\}=\{011\}$ and $\left\{R_{R T F=1}, I_{R T F=1}, Z I N_{R T F=1}\right\}=\{111\}$. Since the maximum depth of the circuit is 1 , each combinational vector derived for $\mathrm{BCC}$ will be sequentialized to two vectors for time frames 0 and 1, respectively. For time frame 0 , we take the test input of $\left\{R_{R T F=0}, I_{R T F=0}, Z I N_{R T F=0}\right\}$ and apply vector $\{011\}$ to $\{R, I, Z I N\}$. Then for the time frame 1 , we take $\left\{R_{R T F=1}, I_{R T F=1}, Z I N_{R T F=1}\right\}$ and apply vector $\{111\}$ to $\{R, I, Z I N\}$. Since copies of $\{R, I, Z I N\}$ exist for both $R T F=0$ and $R T F=1$, sequential vector is fully specified and there is no need to fill random values. Thus, the generated sequential test input for $\{R, I, Z I N\}$ is a two vector sequence, $\{011\}$ and $\{111\}$. Using the GENTEST ATPG system [10], a fault coverage of $100 \%$ was obtained for both original circuit and $\mathrm{BCC}$ shown in Figures 1 and 3.

\section{Results}

We have implemented the algorithm presented in this paper in a $C$ language program called $B C C$. Tables 1 and 2 show the results of experiments on the ISCAS 89 benchmark circuits. For the cyclic ISCAS 89 benchmark circuits, we scanned minimal sets of FFs to make the kernel cir. cuit acyclic. Using improved algorithms, in most cases we: scanned fewer FFs than reported by Min and Rogers [20]. We only present the results of applicable circuits, i.e., acyclic: circuits with at least one unscanned FF. For test generation, we used the GENTEST ATPG system [10] for both combinational and sequential circuits, running on a Sun Ultra II workstation with dual $200 \mathrm{MHz}$ processors.

$\mathrm{BCC}$ test generation was performed as follows. First, we generate combinational tests for all single stuck-at faults of $\mathrm{BCC}$ as a first vector set. The sequentialized first vector set will detect all detectable single-mapped faults and most of multiple-mapped faults in the original acyclic sequential circuit. Then, we use a sequential fault simulator to simulate the sequentialized first vector set. Each fault, not detected by the sequentialized first vector set, must be processed and classified as: (1) an undetectable single-mapped fault, (2) an undetectable multiple-mapped fault, or (3) a detectable multiplemapped fault. This fault classification is unnecessary if the fault coverage (FC) is already $100 \%$.

Table 1 shows the test generation results and gives a comparison between the combinational ATPG using the BCC and the conventional sequential ATPG. Fault Coverage (FC), fault efficiency (FE), test vector length (Vectors), total test generation time (TGT) and averaged test generation time per fault (TPF) for both combinational and sequential ATPG are included in the table, under columns $F C, F E$, Vectors, TGT and $T P F$, respectively. Last two columns show the speed-up ratios of combinational over sequential approaches for TGT and TPF. As expected, the simpler combinationally balancel model ATPG is significantly faster than the sequential ATPG. We observe as much as 5.9 times faster test generation.

It is important to notice that the results are generated using a default time limit set by GENTEST ATPG. Same time limit was used for both combinational and sequential test genera. tion. For circuits with aborted faults, it is possible to increase: the time limit per fault to make the differences in the test gen. eration times more dramatic. In all cases, the new method yielded equal or better fault coverages (FC) and fault efficien. cies (FE) as shown in Table 1. The combinational approach gave a higher fault efficiency for $\mathrm{s} 9234$, as two aborted fault: were detected. Higher fault coverage (one more fault de. tected) was obtained for $\mathbf{3} 38584$ in less time than the sequen. tial ATPG. However, test vector lengths of BCC are much longer than sequential method.

Above results are reported without any vector compaction, but there are several ways to compact the combinationally. derived test vectors. One can compact the $\mathrm{BCC}$ vectors with a simple reverse order simulations and drop the vectors that do not detect new faults. In general, we obtain about $50 \%$ reduction this way. After this set is sequentialized, the se. quence is further compacted using sequential circuit fault simulation. The final number of compacted vectors can bo 
Table 1. Test generation results (Sun Ultra II, 200MHz Dual CPUs): combinational vs. sequential ATPG.

\begin{tabular}{|c|c|c|c|c|c|c|c|c|c|c|c|c|}
\hline \multirow{3}{*}{$\begin{array}{l}\text { Circuit } \\
\text { Name }\end{array}$} & \multicolumn{5}{|c|}{ Combinational ATPG } & \multicolumn{5}{|c|}{ Sequential ATPG } & \multirow{2}{*}{\multicolumn{2}{|c|}{$\begin{array}{c}\text { ATPG } \\
\text { speed-up }\end{array}$}} \\
\hline & \multirow{2}{*}{$\begin{array}{c}\mathrm{FC} \\
\% \\
\end{array}$} & \multirow{2}{*}{$\begin{array}{c}\mathrm{FE} \\
\% \\
\end{array}$} & \multirow[t]{2}{*}{ Vectors } & \multirow{2}{*}{$\begin{array}{c}\text { TGT } \\
\mathrm{s}\end{array}$} & \multirow{2}{*}{$\begin{array}{l}\text { TPF } \\
\mathrm{ms}\end{array}$} & \multirow{2}{*}{$\begin{array}{c}\text { FC } \\
\% \\
\end{array}$} & \multirow{2}{*}{$\begin{array}{c}\mathrm{FE} \\
\%\end{array}$} & \multirow[t]{2}{*}{ Vectors } & \multirow{2}{*}{$\begin{array}{c}\text { TGT } \\
\text { s }\end{array}$} & \multirow{2}{*}{$\begin{array}{c}\text { TPF } \\
\text { ms }\end{array}$} & & \\
\hline & & & & & & & & & & & TGT & TPF \\
\hline $\mathrm{s} 382$ & 100.00 & 100.00 & 116 & 0.10 & 1.72 & 100.00 & 100.00 & 81 & 0.18 & 3.08 & 2.0 & 1.9 \\
\hline$s 400$ & 98.50 & 100.00 & 118 & 0.10 & 1.46 & 98.50 & 100.00 & 83 & 0.13 & 1.73 & 1.4 & 1.3 \\
\hline s444 & 97.52 & 100.00 & 112 & 0.14 & 1.63 & 97.52 & 100.00 & 77 & 0.15 & 1.79 & 1.1 & 1.2 \\
\hline s641 & 100.00 & 100.00 & 196 & 0.22 & 2.20 & 100.00 & 100.00 & 112 & 0.34 & 3.43 & 1.6 & 1.6 \\
\hline s713 & 94.88 & 100.00 & 216 & 0.94 & 5.17 & 94.88 & 100.00 & 118 & 1.02 & 5.76 & 1.2 & 1.2 \\
\hline s953 & 100.00 & 100.00 & 214 & 0.37 & 3.42 & 100.00 & 100.00 & 182 & 0.49 & 4.04 & 1.0 & 0.9 \\
\hline s1196 & 99.87 & 100.00 & 1456 & 1.10 & 2.69 & 99.87 & 100.00 & 304 & 1.33 & 6.47 & 1.2 & 2.4 \\
\hline s1238 & 96.64 & 100.00 & 1532 & 2.15 & 3.86 & 96.64 & 100.00 & 327 & 2.83 & 9.63 & 1.5 & 2.8 \\
\hline $\mathrm{s} 1423$ & 99.08 & 100.00 & 182 & 1.43 & 7.02 & 99.08 & 100.00 & 182 & 2.17 & 11.42 & 1.7 & 1.8 \\
\hline s5378 & 93.69 & 99.71 & 32000 & 293.22 & 108.88 & 93.69 & 99.71 & 1117 & 1268.00 & 1640.36 & 3.9 & 13.7 \\
\hline s9234 & 93.16 & 99.95 & 5460 & 410.21 & 216.01 & 93.16 & 99.94 & 1233 & 425.63 & 337.80 & 1.2 & 1.82 \\
\hline s13207 & 97.13 & 99.97 & 74773 & 417.08 & 85.94 & 97.13 & 99.97 & 2442 & 1008.04 & 739.57 & 2.6 & 9.2 \\
\hline s15850 & 96.65 & 99.97 & 183270 & 146.17 & 12.62 & 96.65 & 99.97 & 2507 & 853.49 & 535.78 & 5.9 & 42.6 \\
\hline s35932 & 89.80 & 100.00 & 258370 & 444.92 & 60.27 & 89.80 & 100.00 & 2377 & 569.07 & 122.01 & 1.5 & 2.3 \\
\hline s38417 & 99.25 & 99.54 & 116550 & 390.49 & 108.38 & 99.25 & 99.54 & 5360 & 860.87 & 358.84 & 2.2 & 3.3 \\
\hline s38584 & 95.66 & 99.96 & 405216 & 5105.90 & 236.01 & 95.65 & 99.95 & 5763 & 7293.11 & 1479.01 & 1.4 & 6.3 \\
\hline
\end{tabular}

Table 2. Circuit statistics.

\begin{tabular}{|c|c|c|c|c|c|c|c|}
\hline \multirow{2}{*}{$\begin{array}{c}\text { Circuit } \\
\text { name }\end{array}$} & \multicolumn{3}{|c|}{ FFs } & \multirow{2}{*}{$\begin{array}{l}\text { Max. } \\
\text { depth }\end{array}$} & \multicolumn{2}{|c|}{$\mathrm{BCC}$ size $(+\%)$} & \multirow{2}{*}{$\begin{array}{c}\mathrm{MF} \\
\%\end{array}$} \\
\hline & Total & Scan & $\operatorname{Scan}(\%)$ & & PI & gate & \\
\hline s382 & 21 & 15 & 71.4 & 1 & 0.0 & 0.0 & 0.0 \\
\hline$s 400$ & 21 & 15 & 71.4 & 1 & 0.0 & 0.0 & 0.0 \\
\hline s444 & 21 & 15 & 71.4 & 1 & 0.0 & 0.0 & 0.0 \\
\hline s641 & 19 & 15 & 78.9 & 1 & 0.6 & 2.0 & 0.0 \\
\hline s713 & 19 & 15 & 78.9 & 1 & 0.6 & 2.0 & 0.0 \\
\hline s953 & 29 & 23 & 79.3 & 1 & 0.0 & 0.0 & 0.0 \\
\hline s1196 & 18 & 0 & 0.0 & 3 & 52.6 & 264.3 & 0.0 \\
\hline s1238 & 18 & 0 & 0.0 & 3 & 54.2 & 264.3 & 0.7 \\
\hline s 1423 & 74 & 71 & 95.9 & 1 & 1.7 & 6.8 & 0.0 \\
\hline s5378 & 179 & 30 & 16.8 & 19 & 226.1 & 778.5 & 2.5 \\
\hline s9234 & 228 & 152 & 66.7 & 4 & 39.9 & 114.4 & 4.2 \\
\hline s13207 & 669 & 310 & 46.3 & 22 & 110.7 & 240.8 & 1.6 \\
\hline s15850 & 597 & 441 & 73.9 & 29 & 243.4 & 627.5 & 1.4 \\
\hline s35932 & 1728 & 306 & 17.7 & 34 & 254.7 & 320.4 & 1.7 \\
\hline s38417 & 1638 & 1080 & 69.9 & 9 & 15.3 & 78.6 & 1.2 \\
\hline s38584 & 1425 & 1115 & 78.2 & 35 & 141.0 & 376.5 & 0.3 \\
\hline
\end{tabular}

similar to the sequentially-derived test length. For 55378 , a 32000 -vector BCC derived sequence reduced to about 16000 vectors when simple reverse-order combinational fault simulation was used. 32000 vectors were reduced to 5384 vectors with sequential fault simulation and to 4236 vectors when both methods were combined. In either case, added CPU time and processing time was less than $10 \%$ of sequential TGT.

Table 2 shows statistics for ISCAS 89 benchmark circuits and their BCC. Three columns under FFs, namely Total, Scan and $\operatorname{Scan}(\%)$, give the total number of FFs, number of scan FFs used to make the circuit acyclic and \% of scan FFs, respectively. The column Max depth shows the maximum sequential depth of acyclic circuits after removing the scan FFs. The increase in numbers of PIs and gates of BCC over the original circuits are shown in two columns, PI and gates un- der the heading $B C C$ size (+\%). The increase in PIs varies from 0 to $250 \%$ and increase in gates between 0 to $778 \%$. In general, circuits with more FFs and larger maximum sequential depth have a lager increase in BCC size than the circuits with fewer FFs and smaller depth. The reader should not mistake the increased BCC size for DFT hardware overhead. The $B C C$ size is only an indicator of the size of the combinational ATPG model circuit.

For some faults, the balanced combinational model has one-to-multiple mapping. These multiple faults correspond to faults at the same site in the original circuit at different time-frames. When the combinational ATPG program is not equipped with multiple fault detection capability, a single fault assumption still produces tests for most faults as we have found. However, we can easily model the multiple faults with a simple modification. A single stuck-at fault on an added signal feeding into two-input OR or AND gates inserted at fault sites represents the multiple stuck-at fault. The test generation time for such multiple-mapped fault is same as any other single fault. The last column in Table 2 shows the \% of multiple-mapped faults (MF) that had to be modeled. We found that most (about 95\%) of the undetected faults did not require multiple fault mapping.

\section{Conclusion}

Our target circuit for test generation is a circuit that is either originally acyclic or has been made acyclic by partial scan or any other DFT method. Our proposed test generation method for any general acyclic sequential circuit requires only a combinational ATPG. Our test generation method is based on transforming the unbalanced acyclic sequential circuit to a fault equivalent combinational circuit model by moving all unbalanced fanouts to PIs and then adding new PIs. 
Added PIs allow combinational model to create non-repeated vectors to detect a fault in the original acyclic circuit. This is significantly different from a previous combinational model [20] where repeated test patterns were used. Our method detects all sequentially detectable faults, and also identifies all sequentially untestable faults to achieve maximum possible fault efficiency faster than the conventional sequential test generation approach. Because a combinational model is used to generate tests, the test generation time spent on detectable as well as undetectable faults is significantly lower.

\section{Future Studies}

Since the realization that feedback in a sequential circuit adds to the test generation complexity, there have been numerous reports $[3,7,8,15,17]$ on reducing the complexity of test generation by breaking cycles. Scan design is a widely used DFT method for breaking loops and cycles in a sequential circuit. Scan DFT can not only make the circuit loop/cycle-free, but can also make the circuit strongly balanced, weakly balanced or leave it unbalanced. However, there has been no research conducted on nor formal algorithms given for selecting scan FFs to make the circuit balanced beyond the work of Gupta et al. [15]. With a proper algorithm, we believe that more compact and efficient acyclic balanced sequential circuit can be obtained.

Similar to scan DFT, the multiple-clock (MC) can also be used to break loops in the circuits [4]. The use of MC DFT to break cycles may not be as straightforward as scan DFT, but it has its advantages. It can break (or block) loops without scanning in and out. We also believe that MC DFT can be used to assist in creating a balanced circuit. With a proper integration of the scan DFT and MC DFT, test generation of sequential circuits may be even more efficient and less costly.

Acknowledgment - Authors thank Arun Balakrishnan for supplying the MFVS for several ISCAS ' 89 circuits.

\section{References}

[1] M. Abramovici, M. A. Breuer, and A. D. Friedman, Digital Systems Testing and Testable Design. New York: IEEE Press, 1990.

[2] V. D. Agrawal, editor, Special Issue on Partial Scan Methods, volume 7 of $J$. Electronic Testing: Theory and Applic. Boston: Kluwer Academic Publishers, Aug.-Oct. 1995. no. 1/2.

[3] V. D. Agrawal, K.-T. Cheng, D. D. Johnson, and T. Lin, "Designing Circuits with Partial Scan," IEEE Design \& Test of Comput., vol. 6, no. 2, pp. 8-15, Apr. 1988.

[4] V. D. Agrawal, S. C. Seth, and J. S. Deogun, "Design for Testability and Test Generation with two clocks," in Proc. 4th Int. Symp. on VLSI Design, Jan. 1991, pp. 44-51.

[5] A. Balakrishnan and S. T. Chakradhar, "Software Transformations for Sequential Test Generation," in The Fourth Asian Test Symp., Nov. 1995, pp. 266-272.
[6] A. Balakrishnan and S. T. Chakradhar, "Sequential Circuits with Combinational Test Generation Complexity," in Proc. 9th International Conference on VLSI Design, Jan. 1996, pp. 111 117.

[7] S. T. Chakradhar, A. Balakrishnan, and V. D. Agrawal, "An Exact Algorithm for Selecting Scan Flip-Flops," J. Electronic Testing: Theory and Applic., vol. 7, no. 2, pp. 71-82, Oct. 1995.

[8] K.-T. Cheng and V. D. Agrawal, "A Partial Scan Method for Sequential Circuits with Feedback," IEEE Trans. Computers, vol. 39, no. 4, pp. 544-548, Apr. 1990.

[9] K.-T. Cheng and V. D. Agrawal, "State Assignment for Testable Design," Int. Journal of Comp. Aided VLSI Design, vol. 3, no. 3, pp. 291-308, 1991.

[10] W. T. Cheng and T. J. Chakraborty, "GENTEST: An Automatic Test Generation System for Sequential Circuits," Computer, vol. 22, no. 4, pp. 43-49, Apr. 1989.

[11] E. B. Eichelberger, E. Lindbloom, J. A. Waicukauski, and T. W. Williams, Structured Logic Testing. Englewood Cliffs, New Jersey: Prentice-Hall, 1991.

[12] H. Fujiwara, "A New Class of Sequential Circuits with Combinational Test Generation Complexity," IEEE Trans. on Com. puters, vol. 49, no. 9, pp. 895-905, Sep. 2000.

[13] H. Fujiwara, "A New Definition and A New Class of Sequential Circuits with Combinational Test Generation Complexity," in Proc. Int. Conf. on VLSI Desigr, Jan. 2000, pp. 288-293.

[14] H. Fujiwara, S. Ohtake, and T. Takasaki, "Sequential Circuit Structure with Sombinational Test Generation Complexity and its Application," Trans. IEICE (DI) (in Japanese), vol. J80-D1, no. 2, pp. 155-163, Feb. 1997.

[15] R. Gupta, R. Gupta, and M. A. Breuer, "The BALLAST Methodology for Structured Partial Scan Design," IEEE Trans. Computers, vol. 39, no. 4, pp. 538-548, Apr. 1990.

[16] M. Inoue, E. Gizdarski, and H. Fujiwara, "Theorems for Separable Primary Input Faults in Internally Balanced Structures," Information Sceince Technical Report, vol. ISSN 0919-9527, no. NAIST-IS-TR2000004, pp. 1-5, Mar. 2000.

[17] A. Kunzmann and H. J. Wunderlich, "An Analytical Approach to the Partial Scan Problem," J. Electronic Testing: Theory and Applic., vol. 1, no. 2, pp. 163-174, Apr. 1990.

[18] C. E. Leiserson and J. B. Saxe, "Retiming Synchronous Circuitry," Algorithmica, vol. 6, no. 1, pp. 5-35, 1991.

[19] A. Miczo, Digital Logic Testing and Simulation. New York: Harper \& Row, 1990.

[20] H. B. Min and W. A. Rogers, "A Test Methodology for Finite State Machines using Partial Scan Design," J. Electronic Testing: Theory and Applic., vol. 3, no. 2, pp. 127-138, May 1992.

[21] T. Takasaki, T. Inoue, and H. Fujiwara, "Paritial Scan Design Methods Based on Internally Balanced Structure," in Proc. Asia and South Pacific Design Automation Conf., Feb. 1998, pp. 211-216.

[22] H. J. Wunderlich, "The Design of Random-Testable Sequential Circuits," in Proc. Fault-Tolerant Comp. Symp., 1989, pp. 110117. 